\title{
Prevalence of Microalbuminuria in NIDDM Hypertensives and Non-Diabetic Hypertensives A Comparative Study
}

\author{
Dr. Matta SreeVani M.D ${ }^{1}$, Dr. G. J. S. L. Latha M.D ${ }^{2}$, \\ Dr. Bezwada Srinivasa Rao M.D. ${ }^{3}$ \\ ${ }^{l}$ Assistant Professor,Department of Biochemistry, Siddhartha Medical College, NTRUHS, Vijayawada, India. \\ ${ }^{2}$ Assistant Professor, Department of Biochmistry, Siddhartha Medical College, NTRUHS, Vijayawada, India. \\ ${ }^{3}$ Associate Professor, Department of Medicine, Siddhartha Medical College, NTRUHS, Vijayawada, India.
}

\begin{abstract}
Urinary albumin excretion is indicative of glomerular permeability with increasing levels of albumin in the urine indicates renal damage. An excretion rate of $<30 \mathrm{mg} /$ day is normoalbuminuria. While an excretion rate $>300 \mathrm{mg} /$ day is macroalbuminuria. Microalbuminuria of 20-200 $\mathrm{\mu g} / \mathrm{min}(30-300 \mathrm{mg} /$ day $)$ is an early indicator of renal injury and predicts the development of proteinuria and progressive renal failure. Microalbuminuria is also an important predictor of cardiovascular events in patients with both insulin dependent diabetes mellitus [IDDM] and non insulin dependent diabetes mellitus [NIDDM]. Urinary albumin excretion depends on permselectivity of the glomerular basement membrane [GBM]. Majority of patients with nephropathy have NIDDM due to higher prevalence of NIDDM (90\%) in general population. Hypertension accelerates rate of decline of renal function and more closely related to higher blood pressure and longer duration. Increased glomerular hydrostatic pressure and increased permeability of GBM are two proposed mechanisms for increased Urinary Albumin Excretion (UAE).

The aim of study is to compare the prevalence of microalbuminuria in non insulin dependent diabetic [NIDDM] hypertensives with non-diabetic hypertensives and to correlate with sex, age, family history, body mass index(BMI), mean arterial pressure(MAP) risk factors. Only type 2 diabetic hypertensives and non diabetic hypertensives were included. IDDM patients and hypertensives on angiotensin converting enzyme inhibitors [ACEI], angiotensin II receptor blockers [ARBS] were excluded from the study. 150 persons were enrolled into the study. 50 non insulin dependent diabetic hypertensives, 50 non-diabetic hypertensives and 50 healthy individuals were included as controls. Urinary Albumin Excretion[UAE] was estimated by particle enhanced Immunoturbidimetric test in $24 \mathrm{hr}$ urinary sample. The study showed higher prevalence of microalbuminuria in NIDDM with HTN group (74\%) than non-diabetic hypertensive group (38\%). Males showed higher prevalence of microalbuminuria $56.75 \%$ in NIDDM with HTN and $68.42 \%$ in hypertensives than females in both groups. The study showed patients in higher age group 50-60yrs had higher prevalence of microalbuminuria $78.37 \%$ in NIDDM with HTN and $63.15 \%$ in non diabetic hypertensives. It has been observed that increasing of age associated with increased prevalence of microalbuminuria. $74 \%$ of patients in NIDDM with HTN and 54\% of patients in non diabetic hypertensives showed positive family history. Higher BMI 25-35 showed higher prevalence of microalbuminuria $67.56 \%$ in NIDDM with HTN and $42 \%$ in HTN group. Higher prevalence of microalbuminuria $74 \%$ in diabetic hypertensives and $38 \%$ in non diabetic hypertensives with MAP ranging between $111-150 \mathrm{mmHg}$. The results were analysed by applying Chi-square test. -value calculated and compared among three groups. P-value $<0.005$ which is highly significant..
\end{abstract}

Keywords: Glomerular basement membrane, Glomerular hydrostatic pressure, Microalbuminuria, Mean arterial pressure, Urinary Albumin Excretion.

\section{Introduction}

Urinary albumin excretion is indicative of glomerular permeability with increasing levels of albumin in the urine indicates renal damage. An excretion rate $<30 \mathrm{mg}$ /day is normoalbuminuria. While an excretion rate of $>300 \mathrm{mg} /$ day is macroalbuminuria. Microalbuminuria of $20-200 \mu \mathrm{g} / \mathrm{min} /(30-300 \mathrm{mg} /$ day $)$ is an early indicator of renal injury and predicts the development of proteinuria and progressive renal failure.

Microalbuminuria is also an important predictor of cardiovascular events in patients with both insulin dependent diabetes mellitus and non insulin dependent diabetes mellitus. Urinary albumin excretion(UAE) depends on permselectivity of the glomerular basement membrane. Microalbuminuria can be due to the consequence of loss of anionic charge of the glomerular advanced glycosylation end products which will bind and neutralize the anionic proteins of the basement membrane leading to an increase in the transmembrane passage of albumin. In type II diabetes mellitus, the amount of urinary albumin excretion correlates with the deposits of advanced glycosylation end products. Recent studies have shown an association between microalbuminuria and impaired glomerular basement membrane charge selectivity even in healthy subjects. The 
increased permeability of the glomerular basement membrane for albumin could be caused by factors such as vascular endothelial growth factor and vascular permeability factor produced by mesangial or endothelial cells. Vascular permeability factor appears to be implicated in the pathogenesis of microalbuminuria and proteinuria in patients with diabetes and in those with glomerulonephritis.

Growing interests in the study of microalbuminuria in essential hypertension has recently emerged. At least two mechanisms that have been proposed for increased urinary albumin excretion in patients with essential hypertension are increased glomerular hydrostatic pressure and increased permeability of the glomerular basement membrane.The relationship between renal function and hypertension in different studies demonstrated the role of renal excretory function on blood pressure regulation and the presence of an increased fluid volume in hypertension. Progressive renal damage caused by hypertension occurs due to transmission of higher systemic pressure to the afferent arterioles which damages the glomerular cells causing glomerulosclerosis. Glomerular hydrostatic pressure is regulated by the relative vasoconstriction-vasodilatation of the afferent and efferent glomerular arterioles. The tone of these arterioles is regulated by different mechanisms. Calcium channel blockers (CCB) appear to preferentially dilate the afferent arterioles whereas angiotensin converting enzyme inhibitors(ACEI), atrial natriuretic peptide(ANP) preferentially dilate the efferent arterioles. While clinical proteinuria is found with a low frequency in patients with essential hypertension but higher prevalence of microalbuminuria has been reported. A wide variability of UAE (Urinary Albumin Excretion) has also been shown among normotensive healthy subjects. In patients with essential hypertension, several studies have shown a significant correlation between UAE and office blood pressure values. In general a better correlation between levels of blood pressure and UAE was observed when continuous ambulatory blood pressure measurements were used instead of occasional blood pressure readings in the office. The increasing interest in the significance of microalbuminuria in essential hypertension derives in large part from the recognition that an increase in UAE is associated with an increased incidence of cardiovascular complications. An attempt has been made to study the prevalence of microalbuminuria. A sample of 50 non insulin dependent diabetic hypertensives and 50 non diabetic hypertensives and 50 controls were selected for this study. Microalbuminuria studied and was estimated by immunoturbidimetric method.

\section{Aim Of Study}

The aim of study is to compare the prevalence of microalbuminuria in NIDDM hypertensives with non-diabetic hypertensives.

\section{Materials and Methods}

Informed consent was taken from the patients in their own language before collecting data. This study was approved by Ethics Committee of Siddhartha Medical College, Vijayawada. Present study enrolled 150 subjects including 50 cases of non insulin dependent diabetic hypertensives (NIDDM $+\mathrm{HTN}$ ), 50 cases of non diabetic hypertensives and 50 healthy individuals as controls. All the patients who were known diabetics and hypertensives with duration more than $10 \mathrm{yrs}$ and in the age group of 35-60 years were included in the study. Patients who are not on ACEI and ARBS were included in the study as these drugs decreases UAE. Age, height,weight and blood pressure were recorded. Microalbuminuria prevalence was studied in these groups correlating with risk factors like age, sex, family history, BMI, MAP (Mean Arterial Pressure). Detailed case history was taken regarding their family history for diabetes and hypertension. All the cases were taken from the GGH; Vijayawada.

\subsection{Inclusion criteria:}

3.1.1. Age : 35 - 60 Yrs ( age group selected as NIDDM more prevalent after 35 yrs age and to exclude IDDM which occurs at younger age )

3.1.2. Diabetic hypertensives and Non-diabetic hypertensives who are not on ACE inhibitors, ARBS as these drugs tend to decrease UAE.

\subsection{Exclusion criteria:}

\subsubsection{Type I Diabetes ( IDDM )}

3.2.2. Macroscopic proteinuria on routine examination

3.2.3. Older age group $>60$ Yrs, Urinary tract infection, recent intercurrent illness, pregnant women, renal diseases like nephritic syndrome, exercise, sustained erect posture tend to increase urinary albumin excretion.

3.2.4. Patients who are on ACE inhibitors \& ARBS as these drugs tend to decreases UAE.

3.3. Collection of Samples:

3.3.1. Estimation of microalbumin levels in $24 \mathrm{hr}$ urine sample by Particle enhanced immunoturbidimetric test. Urine sample collected immediately after rising as physical activity increases albumin excretion. 
Method::Particle enhanced immunoturbidimetric test (EURO Diagnostic Systems ) [1,2,3]. Priniciple : Microalbumin turbilatex is a quantitative turbidimetric test for the measurement of microalbumin in human urine. Total microalbumin excreted $/ 24 \mathrm{hrs}=$ Protein Concentration $\mathrm{mg} / \mathrm{ltr}$ X volume urine in $24 \mathrm{hrs}$ in liters. Normal Value of microalbumin $=3-30 \mathrm{mg} /$ day.

3.3.2. Estimation of plasma glucose levels: $5 \mathrm{ml}$ of venous blood collected in plain bottle and serum separated. Fasting blood sugars [FBS] and Post prandial blood sugars [PPBS] were estimated by GOP-POD Method By Spectrophotometer $[\mathbf{4 , 5 , 6 ]}$. Normal Values: FBS [95-125mg/dl], PPBS [140-199mg/dl].

$$
\text { Glucose Concentration in mg / } \mathrm{dl}=\frac{\text { Absorbance of Test }}{-- \text { Absorbance of Standard }^{-}}
$$

3.3.3. Mean Arterial Pressure [M A P] was calculated by formula

M A P $=$ DP + 1/3 PP (SP-DP ). SP denotes systolic pressure, DP denotes diastolic pressure, PP denotes pulse pressure which is the difference between SP \& DP. Normal MAP is $70-110 \mathrm{mmHg}$.

3.3.4. Body Mass Index [B M I] [7] calculated in both men and women as follows:

$\mathrm{BMI}=$ Weight in $\mathrm{Kg} /$ Height in meters $^{2}$. Normal Value $=18.5-24.9$

\section{Results}

The present study enrolled total 150 individuals. 50 patients were non insulin dependent diabetic hypertensives, 50 were non diabetic hypertensive patients and 50 were normal subjects as controls. The results were shown in tables and figures given below. The results were analysed by applying Chisqare test. P- value calculated and compared among three groups. P- value $<0.005$ which is highly significant.

Table:1 Microalbuminuria Positivity In The Study

\begin{tabular}{|c|c|c|c|c|c|c|}
\hline Microalbuminuria & \multicolumn{2}{|c|}{ Control } & \multicolumn{2}{c|}{ Niddm+Htn } & \multicolumn{2}{|c|}{ Htn } \\
\hline & $\mathbf{n}$ & $\mathbf{\%}$ & $\mathbf{n}$ & $\mathbf{\%}$ & $\mathbf{N}$ & $\mathbf{\%}$ \\
\hline$<\mathbf{3 0} \mathbf{~ m g / d a y}$ & 50 & $100 \%$ & 13 & $26 \%$ & 31 & $62 \%$ \\
\hline$>\mathbf{3 0} \mathbf{~ m g} /$ day & 0 & $0 \%$ & 37 & $74 \%$ & 19 & $38 \%$ \\
\hline Total & $\mathbf{5 0}$ & $\mathbf{1 0 0} \%$ & $\mathbf{5 0}$ & $\mathbf{1 0 0} \%$ & $\mathbf{5 0}$ & $\mathbf{1 0 0 \%}$ \\
\hline
\end{tabular}

Table: 1 above shows distribution of patients with microalbuminuria positivity among three groups in the study. Out of 50 non insulin dependent diabetic hypertensives studied, 37 patients were microalbuminuria positive and out of 50 non diabetic hypertensives, 19 patients were microalbuminuria positive. Out of 50 normal individuals in control group, microalbuminuria positivity was nil.

Fig: 1 below shows percentage of microalbuminuria positivity in the study. $74 \%$ of patients in NIDDM+HTN , $38 \%$ of patients in HTN group showed positive for microalbuminuria. None of subjects in control group were positive for microalbuminuria.

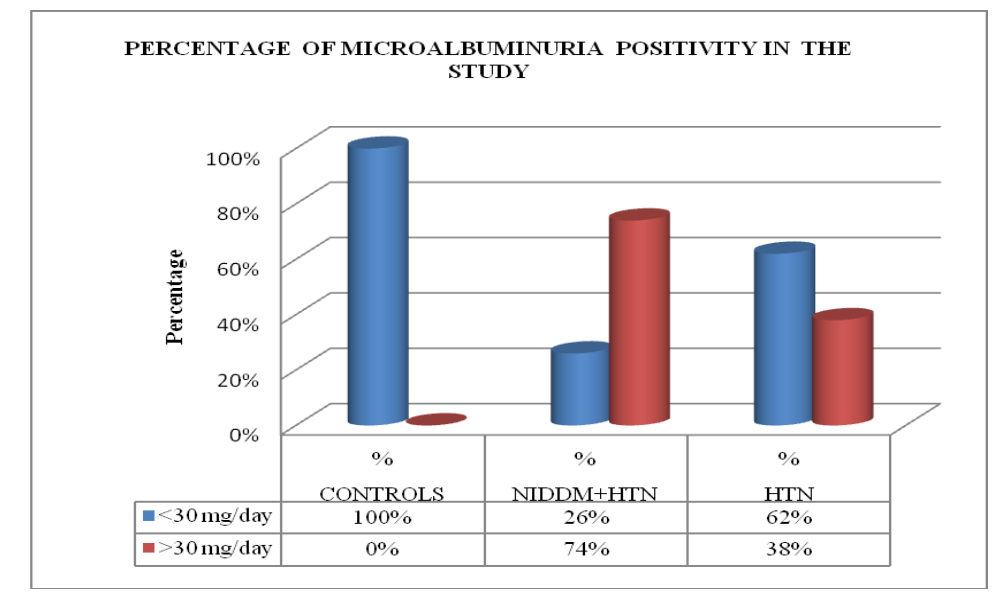

Fig:1

Controls when compared with NIDDM + HTN $\quad---\quad \quad \chi 2=58.73, \quad \mathrm{P}<0.005 \quad---$ Significant

Controls when compared with Non-Diabetic Hypertensives --- $\chi 2=23.46, \mathrm{P}<0.005$--- Significant

NIDDM + HTN compared with Non- Diabetic Hypertensives --- $\chi 2=13.1494, \quad \mathrm{P}<0.005$---Significant 
The sex wise distribution of patients in the study is shown below in Table: 2

Table:2 Sex Wise Distribution Of Patients

\begin{tabular}{|c|c|c|c|c|c|}
\hline & FEMALE & $\%$ & MALE & $\%$ & TOTAL \\
\hline CONTROLS & 22 & $44 \%$ & 28 & $56 \%$ & 50 \\
\hline NDDM+HTN & 23 & $46 \%$ & 27 & $54 \%$ & 50 \\
\hline HTN & 21 & $42 \%$ & 29 & $58 \%$ & 50 \\
\hline
\end{tabular}

The prevalence of microalbuminuria more in males when compared to females. In this study, in NIDDM with HTN group, out of 37 patients positive for microalbuminuria 21 were males $(56.75 \%) \& 16$ were females $(43.24 \%)$ and in HTN group, out of 19 patients positive for microalbuminuria, 13 were males (68.42\%), 6 were females(31.57\%) as shown below in Table:3 \& Fig:2

Table:3 Sex Wise Positivity Of Microalbuminuria

\begin{tabular}{|c|c|c|c|c|c|c|}
\hline $\begin{array}{c}\text { MICRO } \\
\text { ALBUMINURIA }\end{array}$ & \multicolumn{2}{|c|}{ CONTROLS } & \multicolumn{2}{c|}{ NIDDM+HTN } & \multicolumn{2}{c|}{ HTN } \\
\hline & FEMALE & MALE & FEMALE & MALE & FEMALE & MALE \\
\hline$>\mathbf{3 0 m g} /$ day & $0(0 \%)$ & $0(0 \%)$ & $16(43.24 \%)$ & $21(56.75 \%)$ & $6(31.57 \%)$ & $13(68.42 \%)$ \\
\hline$<\mathbf{3 0 m g} /$ day & $22(44 \%)$ & $28(56 \%)$ & $7(53.84 \%)$ & $6(46.15 \%)$ & $15(48.38 \%)$ & $16(51.61 \%)$ \\
\hline
\end{tabular}

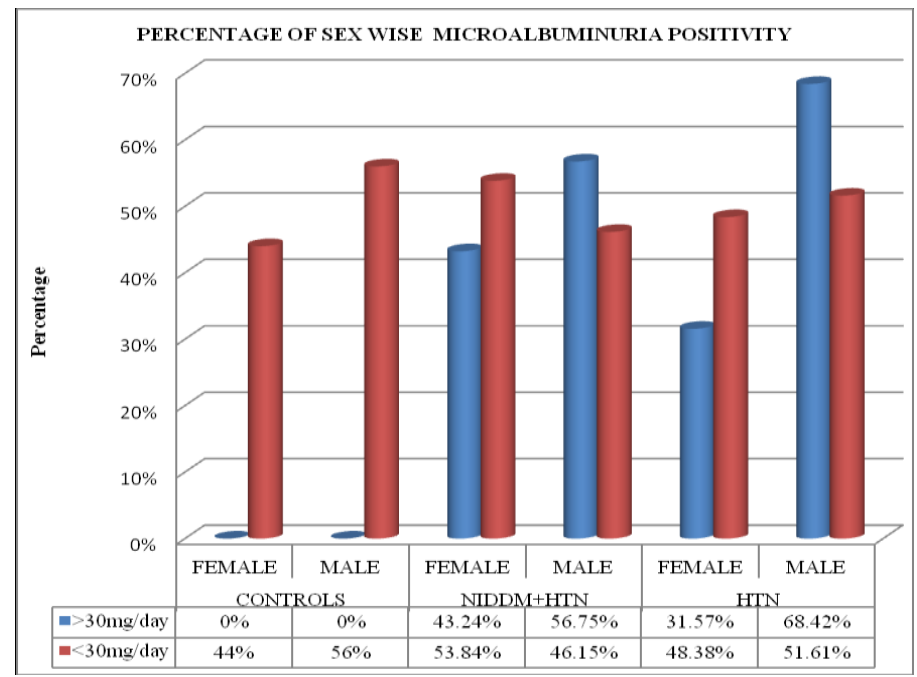

Fig:2

Table : 4 below shows age wise distribution of patients in the study. Majority of the patients included in the study were distributed in the age group between $45-60$ yrs i.e $41(82 \%)$ in diabetic hypertensive group and 37 $(74 \%)$ in hypertensive group. In the age group 45-49yrs, 10 patients $(20 \%)$ in NIDDM+HTN group, 13 patients $(26 \%)$ in HTN group were distributed. The study included $62 \%$ of patients in NIDDM with HTN group and $48 \%$ of non diabetic hypertensives in the age group between 50-60 yrs.

Table:4 Age Wise Distribution Of Patients

\begin{tabular}{|c|c|c|c|c|c|c|}
\hline AGE & \multicolumn{2}{|c|}{ CONTROLS } & \multicolumn{2}{c|}{ NIDDM+HTN } & \multicolumn{2}{c|}{ HTN } \\
\hline & $\mathbf{n}$ & $\%$ & $\mathbf{n}$ & $\%$ & $\mathbf{n}$ & $\%$ \\
\hline $\mathbf{3 5}-\mathbf{3 9}$ & 12 & $24 \%$ & 3 & $6 \%$ & 5 & $10 \%$ \\
\hline $\mathbf{4 0 - 4 4}$ & 11 & $22 \%$ & 6 & $12 \%$ & 8 & $16 \%$ \\
\hline $\mathbf{4 5}-\mathbf{4 9}$ & 14 & $28 \%$ & 10 & $20 \%$ & 13 & $26 \%$ \\
\hline $\mathbf{5 0 - 5 4}$ & 3 & $6 \%$ & 11 & $22 \%$ & 14 & $28 \%$ \\
\hline $\mathbf{5 5}-60$ & 10 & $20 \%$ & 20 & $40 \%$ & 10 & $20 \%$ \\
\hline
\end{tabular}

In NIDDM with HTN group, out of 37 patients positive for microalbuminuria, 29 patients were in $5^{\text {th }}$ decade and in non diabetic hypertensive group, out of 19 patients positive for microalbuminuria 12 
patients were in $5^{\text {th }}$ decade. The least age individual who has microalbuminuria in our study was $35 \mathrm{yrs}$. It has been observed that increase in age was associated with increased prevalence microalbuminuria as per Table:5 given below.

Table:5 Age Wise Positivity Of Microalbuminuria

\begin{tabular}{|c|c|c|c|c|c|c|}
\hline AGE & \multicolumn{2}{|c|}{ CONTROLS } & \multicolumn{2}{c|}{ NIDDM+HTN } & \multicolumn{2}{c|}{ HTN } \\
\hline & $>\mathbf{3 0 m g} /$ day & $<\mathbf{3 0 m g / d a y}$ & $>\mathbf{3 0 m g} /$ day & $<\mathbf{3 0 m g / d a y}$ & $>\mathbf{3 0 m g} /$ day & $<\mathbf{3 0 m g / d a y ~}$ \\
\hline $\mathbf{3 5 - 3 9}$ & $0(0 \%)$ & $12(24 \%)$ & $1(2.70 \%)$ & $2(15.38 \%)$ & $0(0 \%)$ & $5(16.13 \%)$ \\
\hline $\mathbf{4 0 - 4 4}$ & $0(0 \%)$ & $11(22 \%)$ & $2(5.40 \%)$ & $4(30.76 \%)$ & $3(15.78 \%)$ & $5(16.13 \%)$ \\
\hline $\mathbf{4 5 - 4 9}$ & $0(0 \%)$ & $14(28 \%)$ & $5(13.50 \%)$ & $5(38.46 \%)$ & $4(21.05 \%)$ & $9(29.03 \%)$ \\
\hline $\mathbf{5 0 - 5 4}$ & $0(0 \%)$ & $3(6 \%)$ & $10(27.02 \%)$ & $1(7.69 \%)$ & $6(31.57 \%)$ & $8(25.80 \%)$ \\
\hline $\mathbf{5 5 - 6 0}$ & $0(0 \%)$ & $10(20 \%)$ & $19(51.35 \%)$ & $1(7.69 \%)$ & $6(31.57 \%)$ & $4(12.90 \%)$ \\
\hline
\end{tabular}

Fig: 3 reveals percentage of age wise microalbuminuria positivity. The study showed higher prevalence of microalbuminuria with increasing age. $78.37 \%$ in NIDDM+HTN group and $63.15 \%$ in non diabetic group had higher prevalence in $5^{\text {th }}$ decade.

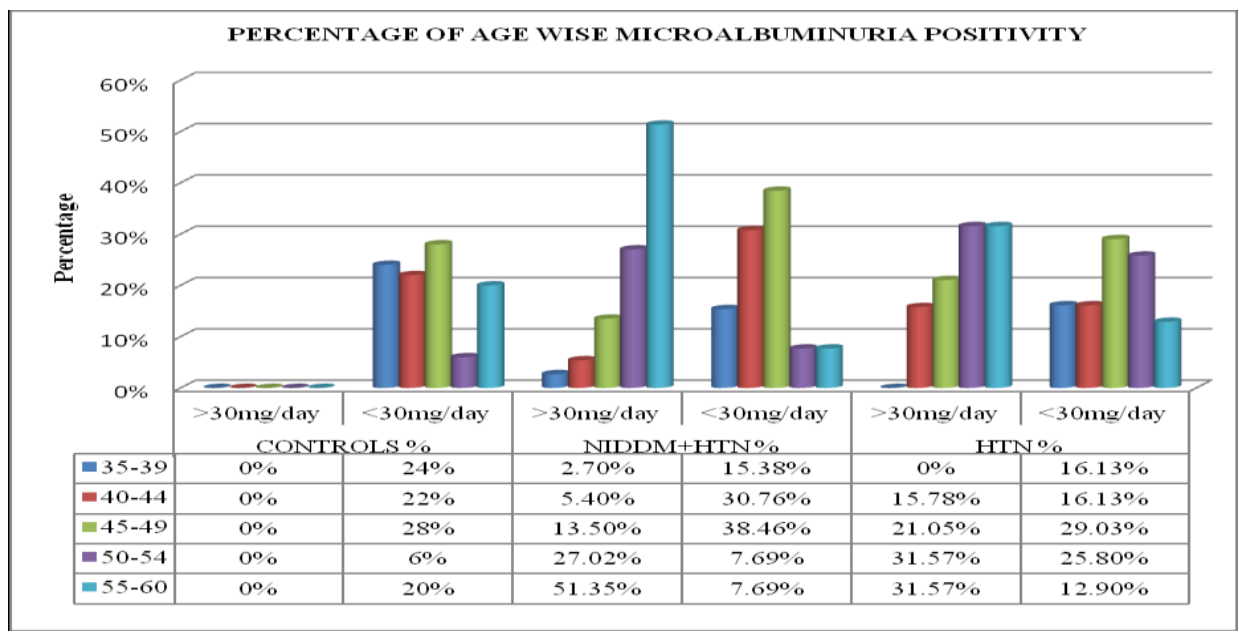

Fig:3

In this study, detailed family history regarding diabetes and hypertension were taken. In diabetic hypertensive group out of 50 patients included 37 patients (74\%) had positive family history, 13 patients $(26 \%)$ had negative family history, In HTN group out of 50 patients ,27 patients (54\%) had positive family history, 23 patients $(46 \%)$ had negative family history. In controls out of 50 patients, 17 patients (34\%) had positive family history, 33 patients $(66 \%)$ had negative family history as per Table:6 shown below.

Table:6 Positive Family History In The Study

\begin{tabular}{|c|c|c|c|c|c|c|}
\hline Family History & \multicolumn{2}{|c|}{ Controls } & \multicolumn{2}{c|}{ Niddm+Htn } & \multicolumn{2}{c|}{ Htn } \\
\hline & Female & Male & Female & Male & Female & Male \\
\hline POSITIVE & $7(14 \%)$ & $10(20 \%)$ & $16(32 \%)$ & $21(42 \%)$ & $10(20 \%)$ & $17(34 \%)$ \\
\hline NEGATIVE & $15(30 \%)$ & $18(36 \%)$ & $7(14 \%)$ & $6(12 \%)$ & $11(22 \%)$ & $12(24 \%)$ \\
\hline
\end{tabular}

In this study the prevalence of microalbuminuria was observed in relation to body mass index [BMI]. In diabetic hypertensives out of 37 patients positive for microalbuminuria, 25 patients $(67.56 \%)$ were in range of 25-35 BMI and 12 patients (32.43\%) were in BMI range 15-25.In hypertensive group, out of 19 patients positive for microalbuminuria, 8 patients $(42 \%)$ were in BMI range $25-35$ and 11 patients $(57.89 \%)$ were in BMI range 15-25. Study showed increased positivity of microalbuminuria in patients with B M I above 25 as shown in Table:7 \& Fig:4 given below.

Table:7 Microalbuminuria Positivity In Relation To Body Mass Index

\begin{tabular}{|c|c|c|c|c|c|c|c|c|}
\hline $\begin{array}{c}\text { Micro } \\
\text { Albuminuria }\end{array}$ & \multicolumn{3}{|c|}{ BMII 15-25 } & \multicolumn{4}{c|}{ BMII 25-35 } \\
\hline & \multicolumn{2}{|c|}{ Niddm+Htn } & \multicolumn{2}{|c|}{ Htn } & Niddm+Htn & \multicolumn{2}{c|}{ Htn } \\
\hline & Female & Male & Female & Male & Female & Female & Male \\
\hline$>30 \mathrm{mg} /$ day & $5(13.51 \%)$ & $7(18.91 \%)$ & $4(21.05 \%)$ & $7(36.84 \%)$ & $12(32.43 \%)$ & $13(35.13 \%)$ & $3(15.78 \%)$ & $5(26.31 \%)$ \\
\hline $\mathbf{3 0 m g} /$ day & $4(30.76 \%)$ & $3(23.07 \%)$ & $13(41.93 \%)$ & $15(48.38 \%)$ & $2(15.38 \%)$ & $4(30.76 \%)$ & $1(3.22 \%)$ & $2(6.45 \%)$ \\
\hline
\end{tabular}




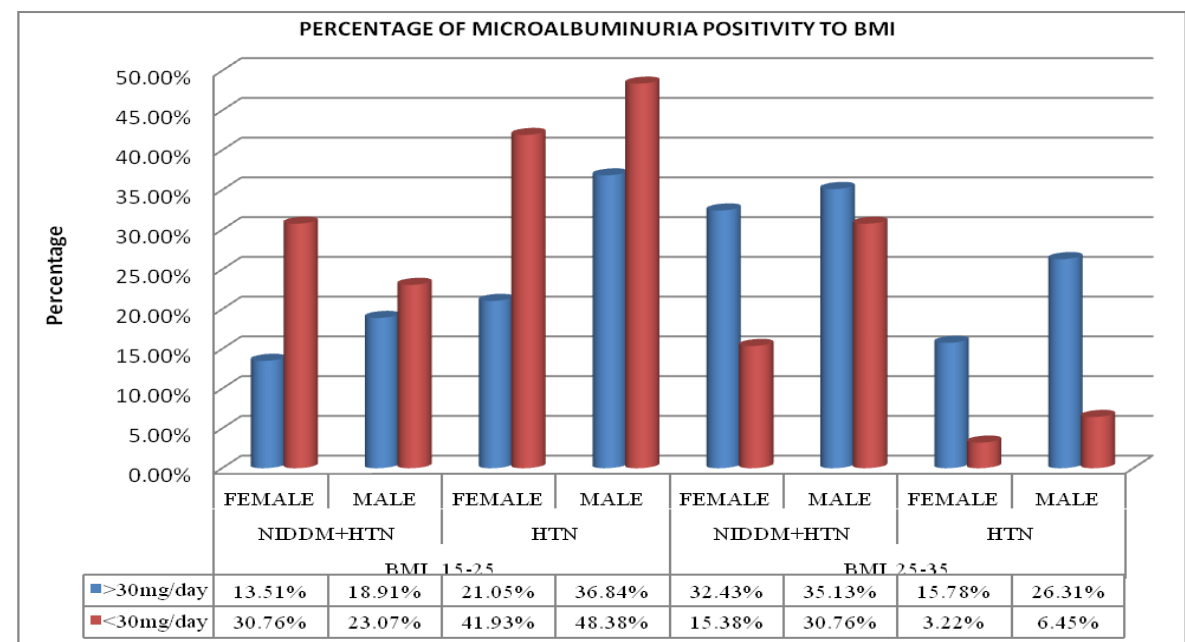

Fig:4

In both groups all the patients had MAP in the range of $111-150 \mathrm{mmHg}$ and in the control group MAP in the range $70-110 \mathrm{mmHg}$ as they were all non hypertensives. In diabetic hypertensive group $74 \%$ patients were microalbuminuria positive, out of which $16(43.24 \%)$ patients were females and $21(56.75 \%)$ patients were males. In hypertensive group 38\% patients were microalbuminuria positive,out of which $6(31.57 \%)$ patients were females and $13(68.42 \%)$ patients were males In control group (normal individuals) all were having microalbuminuria $<30 \mathrm{mg} /$ day as per Table:8,Fig:5 given below.

Table:8 Microalbuminuria Positivity In Relation To Mean Arterial Pressure

\begin{tabular}{|c|c|c|c|c|c|c|c|c|}
\hline \multirow[t]{3}{*}{$\begin{array}{c}\text { Micro } \\
\text { Albuminuria }\end{array}$} & \multicolumn{4}{|c|}{ MAP 70-110 mmHg } & \multicolumn{4}{|c|}{ MAP 111-150 mmHg } \\
\hline & \multicolumn{2}{|c|}{ Niddm+Htn } & \multicolumn{2}{|c|}{ Htn } & \multicolumn{2}{|c|}{ Niddm+Htn } & \multicolumn{2}{|c|}{ Htn } \\
\hline & Female & Male & Female & Male & Female & Male & Female & Male \\
\hline$<30 \mathrm{mg} /$ day & $0(0 \%)$ & $0(0 \%)$ & $0(0 \%)$ & $0(0 \%)$ & $7(53.84 \%)$ & $6(46.15 \%)$ & $15(48.38 \%)$ & $16(51.61 \%)$ \\
\hline
\end{tabular}

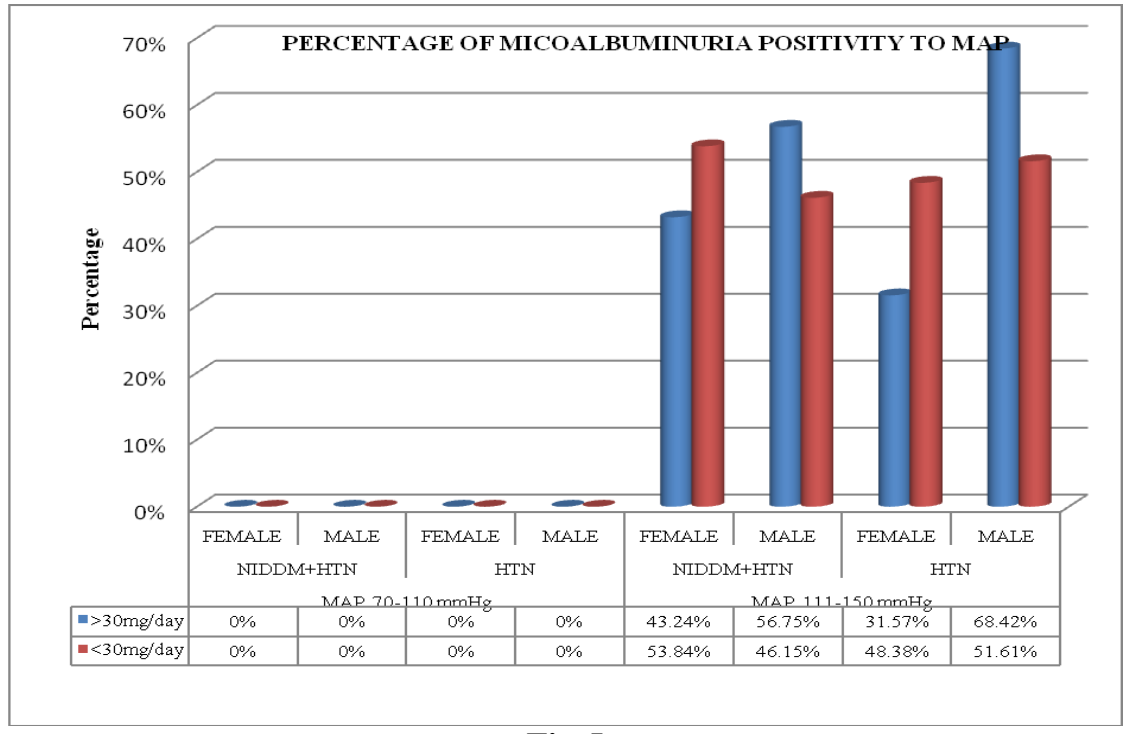

Fig;5

\section{Discussion}

In NIDDM, microalbuminuria is a major independent risk marker for early mortality, the major cause of death being related to cardiovascular disease. Recent studies have shown that high normoalbuminuria also carries a risk. This means that the limits for abnormal albuminuria may have to be revised. Elevated albuminuria in NIDDM may to a large extent, reflect a general vascular leakiness and thus may be a marker of atherosclerosis as well as of diabetic glomerulopathy. This could explain the apparently divergent course of diabetic renal disease seen in NIDDM as compared with IDDM. Studies so far pointed to elevated blood 
pressure and poor glycemic control as factors for worsening progression of albuminuria, decline in kidney function and development of macrovascular disease.

Dr. Maskazu haned[8] and Wiseman M, Viberti G et al[9,10,11] have shown that, in cross-sectional studies in both IDDM and NIDDM, the elevations of blood pressure associated with microalbuminuria. It is, however still controversial whether hypertension is a risk factor for the development of microalbuminuria or results from glomerular damage. The latter hypotheses has been supported by Mathiesen et al[11] who found that the elevation in urinary albumin excretion rates preceded the increase in systemic blood pressure in the patients with IDDM. Pre-existing hypertension may be risk factor for the development of microalbuminuria in subjects with NIDDM[8] A similar conclusion has been reported by Knowler et al[12].

In this study, out of 50 non insulin dependent diabetic hypertensives studied, 37 patients were microalbuminuria positive constituting $74 \%$ and out of 50 non diabetic hypertensives, 19 patients were microalbuminuria positive constituting $38 \%$. Out of 50 normal individuals, microalbuminuria positivity is nil as per Table:1\&Fig:1. This prevalence correlating with the studies carried out by Roberto Bigazzie et al[13]. upto $40 \%$. P value $<0.005$ which is highly significant. According to Keane WF [14]prevalence of microalbuminuria is variable in individuals with hypertension based on age, sex, race between $17-40 \%$.

The sex wise distribution of patients in the study were $27(54 \%)$ males, $23(46 \%)$ females in diabetic hypertensive group ratio being male : female ratio=1.17:1 Hypertensive group constituted $29(58 \%)$ males ,21 $(42 \%)$ females. male : female ratio=1.38:1 as per Table:2. The prevalence of microalbuminuria more in males when compared to females in our study. In NIDDM with HTN group, out of 37 patients positive for microalbuminuria 21 were males $(56.75 \%)$ \& 16 were females $(43.24 \%)$ and in HTN group, out of 19 patients positive for microalbuminuria, 13 were males $(68.42 \%)$, 6 were females $(31.57 \%)$ as per Table:3\&Fig:2. Microalbuminuria prevalence more in males when compared to females as in this study was supported by magic study Pontremoli $\mathrm{R}$ et al[15]. The explanation for this being hormonal protection by estrogen in females.

Majority of the patients included in the study were distributed in the age group between 45-60 yrs i.e $41(82 \%)$ in diabetic hypertensive group and $37(74 \%)$ in hypertensive group. In the age group 45-49yrs, 10 patients $(20 \%)$ in NIDDM+HTN group, 13 patients $(26 \%)$ in HTN group were distributed. The study included $62 \%$ of patients in NIDDM with HTN group and $48 \%$ of non diabetic hypertensives in the age group between 50-60 yrs as per Table:4. It has been observed that increasing of age associated with increased prevalence of microalbuminuria. In NIDDM with HTN group, out of 37 patients positive for microalbuminuria, 29 patients $(78.37 \%)$ were in $5^{\text {th }}$ decade and in non diabetic hypertensive group, out of 19 patients positive for microalbuminuria 12 patients $(63.15 \%)$ were in $5^{\text {th }}$ decade. The least age individual who has microalbuminuria in this study was $35 \mathrm{yrs}$ as shown in Table:5\&Fig:3. It has been observed that increase in age was associated with increased prevalence microalbuminuria. This correlates with study carried out by David Isler Victoria 1995[16].

In this study, detailed family history regarding diabetes and hypertension were taken. In diabetic hypertensive group out of 50 patients included 37 patients $(74 \%)$ had positive family history, 13 patients $(26 \%)$ had negative family history, In HTN group out of 50 patients ,27 patients (54\%) had positive family history, 23 patients $(46 \%)$ had negative family history. In controls out 0 f 50 patients, 17 patients $(34 \%)$ had positive family history, 33 patients $(66 \%)$ had negative family history as shown in Table:6.

In this study the prevalence of microalbuminuria was observed in relation to body mass index [BMI]. In diabetic hypertensives out of 37 patients positive for microalbuminuria,25 patients $(67.56 \%)$ were in range of 25-35 BMI which includes 12 females, 13 males and 12 patients (32.43\%) were in BMI range 15-25 which includes 5 females and 7 males.In hypertensive group, out of 19 patients positive for microalbuminuria, 8 patients $(42 \%)$ were in BMI range 25-35 which includes 3 females, 5 males and 11 patients $(57.89 \%)$ were in BMI range 15-25 which includes 4 females and 7 males as per Table:7\&Fig:4. Study showed increased positivity of microalbuminuria in patients with B M I above 25 . This goes supportive to the study carried out by Metcalf et al[17].

In this study it was observed that severity of hypertension was directly proportional to microalbuminuria. The early screening of hypertension was not existing and hypertension being asymptomatic not making individuals aware of this silent killer. MAP calculated by formula MAP $=$ DP $+1 / 3$ PP (SP-DP). SP denotes systolic pressure, DP denotes diastolic pressure, PP denotes pulse pressure which is the difference between SP \& DP. Normal MAP is $70-110 \mathrm{mmHg}$. As Mean Arterial Pressure (MAP) denotes average blood pressure,MAP calculated and its relation with prevalence of microalbuminuria was observed. In both groups all the patients had MAP in the range of $111-150 \mathrm{mmHg}$ and in the control group MAP in the range $70-110 \mathrm{mmHg}$ as they were all non hypertensives. In diabetic hypertensive group $74 \%$ patients were microalbuminuria positive, out of which $16(43.24 \%)$ patients were females and 21(56.75\%) patients were males.In hypertensive group 38\% patients were microalbuminuria positive, out of which $6(31.57 \%)$ patients were females and 13(68.42\%) patients were males as shown in Table:8\&Fig:5. Roberto Bigaggi et al[13]. found in their study 
that there was no correlation between levels of mean arterial pressures and urinary albumin excretion . Studies carried by Vito M Campese state that the diastolic pressures and diurnal variation of blood pressures influence microalbuminuria. $>300 \mathrm{mg}$ /day of urinary albumin excretion (UAE) was said to be overt nephropathy. Even though $30 \mathrm{mg} /$ day came positive, more the titer positivity more the risk of cardiovascular mortality and renal morbidity[18]. In control group all were having albuminuria $<30 \mathrm{mg} /$ day.

Evaluation of microalbuminuria may be useful in treating diabetes mellitus and hypertensive patients and decreasing cardiovascular, renal morbidity and mortality. ACE inhibitors will reduce proteinuria even with slightest reduction of systolic blood pressures compared to anti-hyperttensives such as calcium channel blockers, $\beta$-blockers, $\alpha$-blockers in Miraten study[18]. From this study it is conceived that microalbuminuria can be used as a predictor of future cardiovascular events and mortality in both diabetics and hypertensive patients.

\section{Conclusion}

Urinary Albumin Excretion is indicative of glomerular permeability with increasing levels of albumin in urine indicates renal damage. Microalbuminuria is an early indicator of renal damage and predicts the development of overt proteinuria. Multiple databases have demonstrated that microalbuminuria is a potent risk factor for the development of progressive renal damage. Microalbuminuria is also an important predictor of cardiovascular events in both NIDDM and IDDM. Several studies have shown significant correlation between UAE and high blood pressure. The aim of study is to compare prevalence of microalbuminuria in both groups. The study showed higher prevalence microalbuminuria in both hypertensives and diabetic hypertensives. Strict control of diabetes and lowering BP with ACEI,ARBS is clearly beneficial in reducing proteinuria, improving renal outcomes and preventing the progression to ESRD. More extensive population based follow up studies are still needed to identify the importance of the present study.

\section{References}

[1]. Feldt-Rasmussen B et al. J Diab Comp 1994; 8: 137-145.

[2]. Medcalf E A et al. Clin Chem 1990; 36/3:446-449.

[3]. Gilbert R et al. Diabrtic Medicine 1994;11:636-645.

[4]. Trinder. P.Ann. Clin.Biochem. 6(24) 1969

[5]. Penncock. C.A. et.al. Clin. Chem.Acto. 48(193) 1973

[6]. Teitz,N.W.Fundamentals of clinical chemistry (243), W.B. Saunders\&Co. Philidelphia PA 1976

[7]. Lippincotts illustrated reviews $3{ }^{\text {rd }}$ Edition Chap 26 page 347

[8]. Dr. Maskazu Haned, Ryvichi kikkawa, masaki Togawa, Daisuke koya, Nobuyuki Kajiwara, Takashi UZU, Yukio shigeta shiga 52021. J of Diabetes.

[9]. Wiseman M, Viberti G. Mackintosh D, Jarrett RJ, Keen H. Glycemia, arterial pressure and microalbuminuria in Diabetes mellitus. Diabetologia 26; 401-405.

[10]. Mogensen CE, Christensen CK; predicting diabetic nephropathy in Diabetes mellitus. N Engl J.Med. 311 : 89-93, 1984.

[11]. Mathiesen ER, Ronn B, Jensen T, Strom B, Deckert T; Relationship between blood pressure and UAE in development of microalbuninuria. Diabetes 39:245.

[12]. Knowler WC, Bennett PH, Nelson RG; Prediabetic blood pressure predicts albuminnuria after development of NIDEDM; Diabetes 37 (supl.1); 120A.

[13]. Roberto Bigazzie et al; prevalence of microalbuminuria in a large population of patients with wild to moderate hypertension Nephron 1992;61;94-97.

[14]. Keane, W.F. et.al; prevalence of Microalbuminuria in Different Races Journal of Nephrology. 622-624.

[15]. Pontremoli R et al : Microalbuminuria a marker of cardiovascular risk and organ damage in essential hypertension. Kidney int .suppl. Dec-63 : S 163-5

[16]. David Iser, Victoria 1995, Thesis on Microalbuminuria Pub-med. Query (Internet).

[17]. Metcalf $\mathrm{P}$ et el : Albuminuria in people atleast $40 \mathrm{yrs}$ old : Effect of obesity, hypertension and hyperlipidaemia. $38 ; 1802-8$.

[18]. Miraten study - microalbuminuria effect of Ramipril and Atenolol, Dr. maneul Luque Madrid. 